\title{
A New Model for Image Segmentation Based on Deep Learning
}

\author{
https://doi.org/10.3991/ijoe.v17i07.21241 \\ Rafeek Mamdouh (四) \\ BADR University, Cairo, Egypt \\ Mansoura University, Mansoura, Egypt \\ rafeekmty@hotmail.com \\ Nashaat El-Khamisy \\ Sadat Academy, Cairo, Egypt \\ Khaled Amer \\ Military Medical Academy, Cairo, Egypt
}

Alaa Riad, Hazem M. El-Bakry

Mansoura University, Mansoura, Egypt

\begin{abstract}
Image segmentation is main point in computer vision (CV) and image processing (IP), that are used routinely in the fields of medicine and surgery training tools. Segmenting images and converting into a model that depends on work by the different algorithms from analysis DICOM files to convert to three-dimensional models. This paper describes a combination of two fields of solving segmentation problem to convert through the workflow of a hybrid algorithm structure Convolutional neural network $(\mathrm{CNN}$, Active Contour \& Deep Multi-Planar) based on seg3d2 to switch DICOM medical rays "Digital Imaging and Communications in Medicine" into a 3Dimintional model, using data from active contour to be the input of deep learning. the result of the pre-processing from DICOM raw images, each image contains edges and image size $=256 \times 256$ pixel, which through adjustment and control we can create multiple results for output using Active Contour, by resizing the threshold frames and gray-scale image, and show liver 3D-model Deep architecture, it is through the CNN which the images of the three axes $\mathrm{X}, \mathrm{Y}$, and $\mathrm{Z}$ (three orthogonal) (coronal $=\mathrm{X}$, sagittal $=\mathrm{Y}$, axial $=\mathrm{Z}=1$ ) are determined and matched with a real image of the body, the area required to be determined, and edits the contrast using a histogram. This research will be using are human liver DICOM images and is divided into two stages (medical image segmentation - retinal model optimization), to help surgeons to study the patient's condition with accuracy and efficiency through the use of mixed reality technology in liver surgery [living donor liver transplantation (LDLT)], all implement by Seg3D2 and Python.
\end{abstract}

Keywords - Hybrid Algorithm, Convolutional neural network (CNN), Active Contour Model (ACM), Digital Imaging and Communications in Medicine (DICOM), living donor liver transplantation (LDLT), histogram equalization, Gaussian Equation Medical Image Processing, Image Segmentation 


\section{Introduction}

\subsection{DICOM \& Big data classifications}

DICOM files contain a lot of information storage known as BIG DATA, most of which is not required. We need to define the working area (the range of interest within the image) to get useful information. DICOM is closely used by hospitals and surgeons such as Brain and liver surgery clinics. DICOM consists of multi-layers images that are combined through a specific system to show radiograph results.

Big data (BD) [1] is a huge collection of information (either structured, semi-structured, or unorganized) on archival units. Big information creates an incentive to build systems of the store and prepares data that cannot be split using traditional processes [2]. Big information do not containing sample data only the same numbers, dates, and strings. BD is Bulky information, contains a Geographic Information System (GIS) 3D model, medical image information, audio, video, DICOM documents, networking documents, and online history [3]. Despite the huge volume of information stored on the electronic cloud that can reach a petabyte or and Exabyte of information, it is related to the process of stability and stability of information, the extent of its exchange, and the implementation of important activities on time, and the extent to which it is possible to analyze and display information in "DICOM" files with huge parameters and stored inside several Layers [4][5][6].

\subsection{Medical Img.Seg. (Image-segmentation)}

Img.Seg. is a complex part of the pattern recognition system and the main part \& 1 st step in image analysis is one of the most complicated steps in image processing and determines the goodness of the result of the analyses. Img.Seg. is an operation of cutoff an image to various areas, such that each region is similar to else [7] [8]. The segmentation model for monocular images can be expanded to color segmentation by using "RBG" or their conversion (linear/non-linear). Although, global surveys on color image segmentation are few [9]. Analyzed the issue when applying edge and area segmentation mode to color images with synthesis texture. [10]. Segmentation is a pertinent technique in image treatment. Various methods occur in multi-apps. Histograms are found to be very efficient in terms of calculation complexity when compared with other division methods. If identified low, High point is properly and proper threshold is fixed, this technique will show good output results [11] It was also implemented in converting DICOM files into a three-dimensional model. [6].

It can take advantage of a set of elements with high-resolution features, such as pixels, texture, and shape properties. Threshold nodes a very simple algorithm to perform hashing. This threshold optimum can be calculated by simulating a Gaussian normal distribution Equation 1 Figure 1, of two regions of the image and calculating the middle and norm deviations of the picture through classified region interest, edges, and background.[12][13]. 


$$
f(x)=\frac{1}{\sqrt{2 \pi \sigma^{2}}} e-\frac{(x-\mu) 2}{2 \sigma^{2}}
$$

Where, $\mu$ : Is expected or mean value, $\sigma^{2}$ : Distribution data point (variance), When $\sigma>0$.

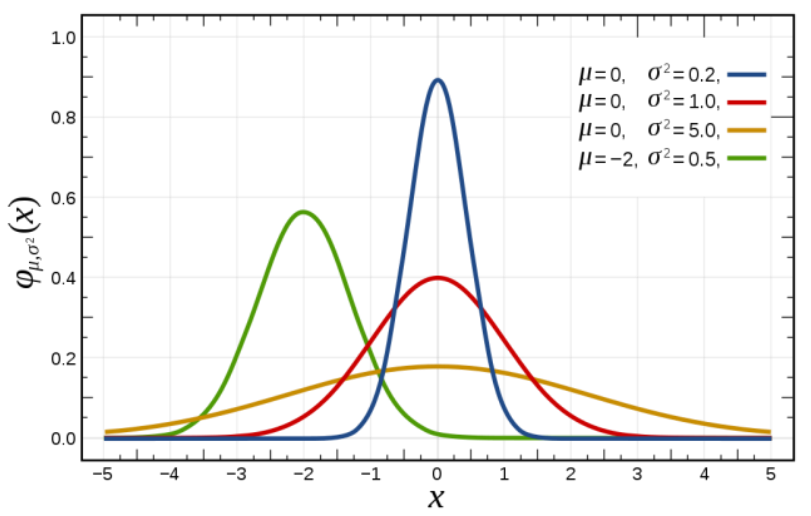

Fig. 1. Red Curve: Normal Distribution Probability Density Functions (PDFs)

\subsection{Anatomical model \& visualization system}

A lot of applications working in surgical and medical image research called Anatomical models in the clinic's the body natural interaction with Virtual-Models simplify anatomy in training and different interactive structures spatially in the human body. Mixed reality is a part of a Visualization system called training Simulation with virtual models anatomy, where to reduces the surgical involvement which is linked to patient risk and cost of healthcare [14]. MR is part of the visualization systems, and it works to create a new reality by integrating a realistic environment with the virtual, which allows the integration of real models and virtual models, to enhance the positive indicators of the surgeon. Applied to liver surgeons during training, before, during, and after the surgical operation in terms of visualization techniques [15]. For example, using Mixed Reality (MR) is an advanced technology used to live in a real environment with fictional models, and it also helps the user to determine its location and medical model location information by Azure Spatial Anchors. Helps classify and store medical images by "Azure Cosmo DB", using Internet of Things services [16]. HOLOLENS devices can live in the real environment and recover $3 \mathrm{D}$ models by improving display speed during training in real-time, by increasing the accuracy of the intensity and degree of illumination while taking into account the degree of illumination distributed in the area to improve the display intensity [17].

\subsection{CNN in medical segmentation}

CNN: "Convolutional-Neural-Network", a part of ANN: "Artificial-Neural-Networks" has been prevailing in different computer vision objectives, benefits across a 
set of domains consist of radiology. CNN designed a spatial hierarchy of features through back-propagation out of a lot of building layers automatically [18]. CNN's hold three types of layers, A) convolution layers, where a kernel of weights is convolved to extract features; B) pooling layers, which replace a small district of a feature map with some statistical input (mean, max, etc.), and C) nonlinear layers, apply an activation duty on feature maps, to can the designing of non-linear duty by the network [19] [20] Figure 2.

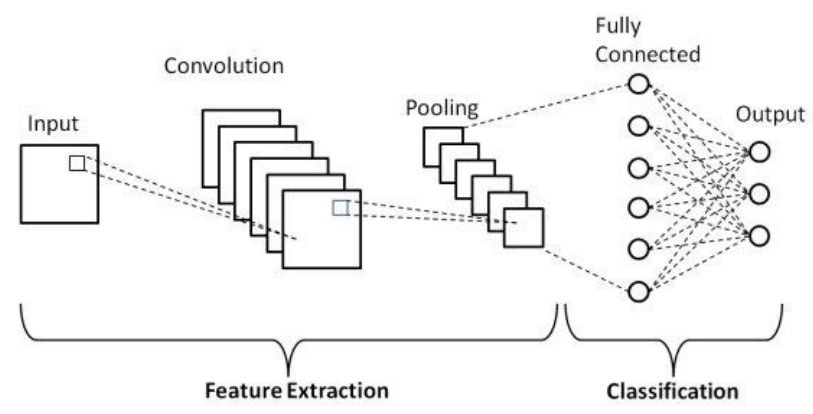

Fig. 2. Diagram Schema of a basic architecture CNN consists of two parts. Feature Extraction: Content 3 layers (Input, Convolution, and pooling layer). Classification: Content 2 layers (Fully Connected and Output layer).

\section{$2 \quad$ Related Work}

The researcher uses part of deep learning called a steady architecture CNN with input trip planar perpendicular patches while be used with advances in convolution kernels and deep networks The fulfillment of totally linked layers as $1 \times 1$ convolution layers and the skip from Shorthand layers pliable rapid diversion of full figures compared with more taking longer timing scanning Moreover Input 3D information patches force result in increased execution performance but require calculation the increased load of the parameter network [21] A new version method using deep learning techniques and dynamic random walker method has been proposed of MR brain image segmentation The hybrid method has shown that best execution as compared through another state methods [22]. The use of CNN and limitations of deep learning and all advantages is primary in a major of radiology research to improve performance radiologists and, patient care [23]. He used an automatic algorithm in liver segmentation to improve the accuracy of the segmentation problem, where it is based on combine between active area (contour) and collect data to use as input for deep learning networks [24]. Using the multi-view and the power of CNN to split, segment PPV and LA through cardiacNET MRI. The method shown, a combination between different information inputs of MRI throw an adaptive combination planning and a new mission, improves damage segmentation and accuracy [25].

He worked on developing a framework to precisely segment all basic structures of the heart from calculating tomography and magnetic chest imaging together with high 
activity. Using multiple CNNs training from the start and permitting an adaptive information fusion strategy in the labeling of pixels was suggested despite data limitations. The results demonstrate a tool to accurately and efficiently identify cardiac structures called [MO-MP-CNN]. Instead of using 3D CNN which is a multi-level 2D CNNs. Thus, use a multi of 2D slides is more important than using a 3Dimentional model within the current setup. In the case of available a lot-of GPU processing and data 3D [26]. The author designed a framework called DMPCT for CT scan segmentation in multiorgan, which is Stimulate by the imitative co-exercise strategy to combine multi-planar input for the dummy data through training, without required a massive of $3 \mathrm{D}$ volumes labor from radiologists [27].

\section{$3 \quad$ Methods}

\subsection{Proposed architecture}

We propose a process to implement liver DICOM image segmentation, and use active contour technique as a method for segmentation as input data where use seg3D2 and CNN network of deep learning Figure 3.

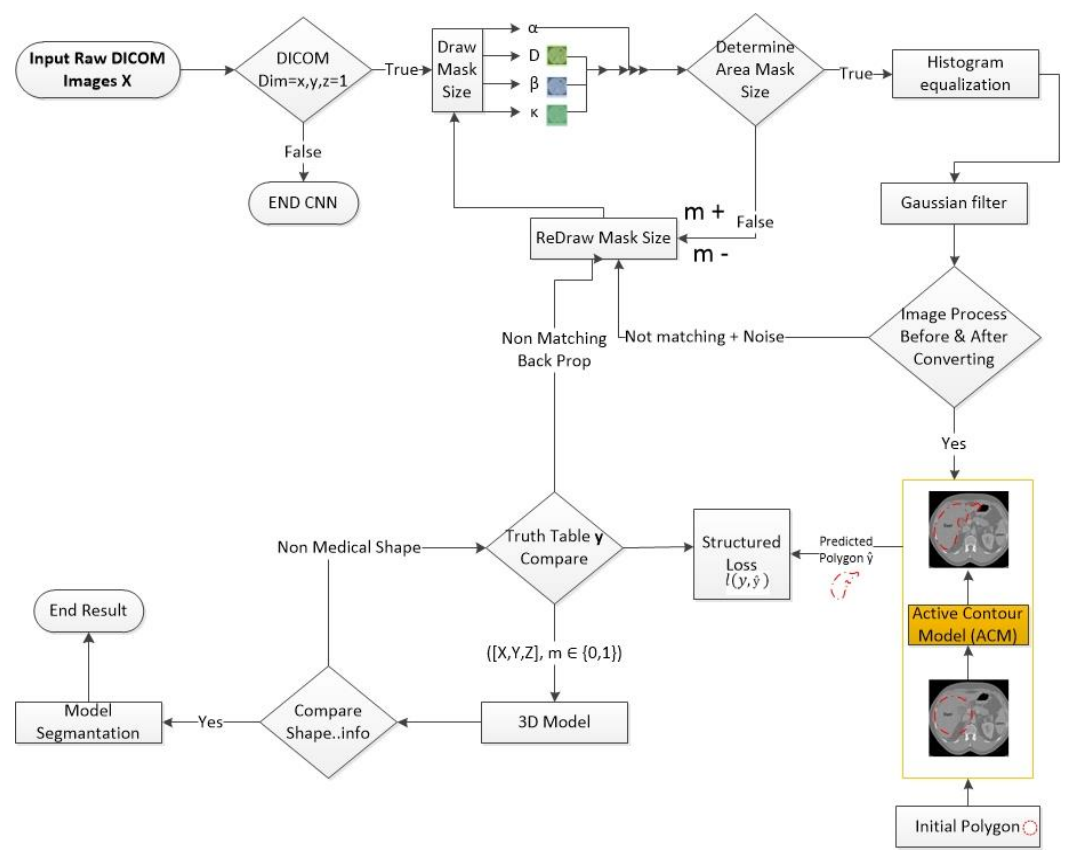

Fig. 3. Flow chart of our proposed DICOM Segmentation and convert to 3D-Model thought seg3D2 and (ACM) mean Active Contour Model, Wherever: a universal $\alpha$ for the extent punishment and local D maps, $\beta$ : the bend penalization, and $\kappa$ : the balloon term. Next step ACM deduction, a structured see is calculated and taken to the CNN, which parameters can already exit updated using back-propagation. 
Since a DICOM image is usually of low contrast, and it is difficult to distinguish between each element and the other and the background, the image must be processed first before starting to split and segment. Due to the large size of the image data and its lack of arrangement, the processing of images plays a large role in defining each element and clarifying the data. As it is the first and required step to improve the quality of the images to obtain high accuracy in reading the data. DICOM images consist of many noises and lighting impurities. Before starting to analyse, segment, and object detection, all impurities must be removed through the processing procedures first.

First of all, we must use the point of convergence and intersection of the DICOM files for the three images (three orthogonal) where (coronal $=X$, sagittal $=Y$, axial $=Z=0$ ) figure 4 as input data and compare them with the 3D model or real images to detect a liver object, Scientific Steps [1]. where the three images are the general shape of the model, and since the images are of low contrast, we should start Convolutional Layer then the histogram equation figure 5 for the raw images must be used as a technique for adjusting the image intensity to improve contrast as shown below figure 6 .

The results show that the images with high noise use the Gaussian Blur technique, while the Gaussian function, in contrast, small and large. We, therefore, recommend Gaussian use, with alpha $=1$.

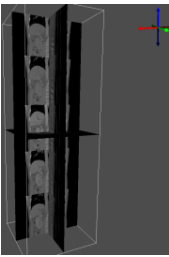

DICOM Volume

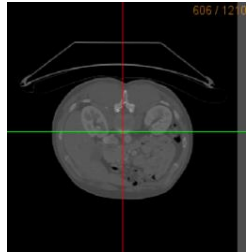

Top: axial=Z

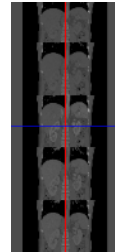

Front: coronal $=\mathrm{X}$

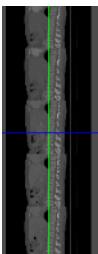

Side: sagittal $=$ Y

Fig. 4. Three Orthogonal

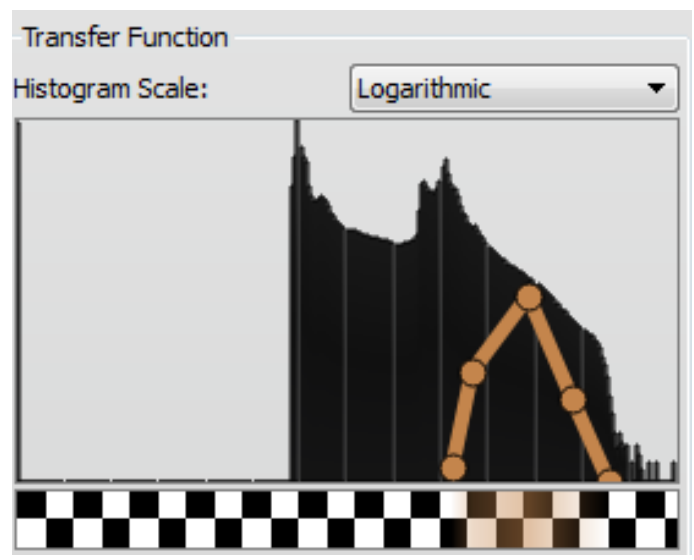

Fig. 5. Data Histogram Transfer 


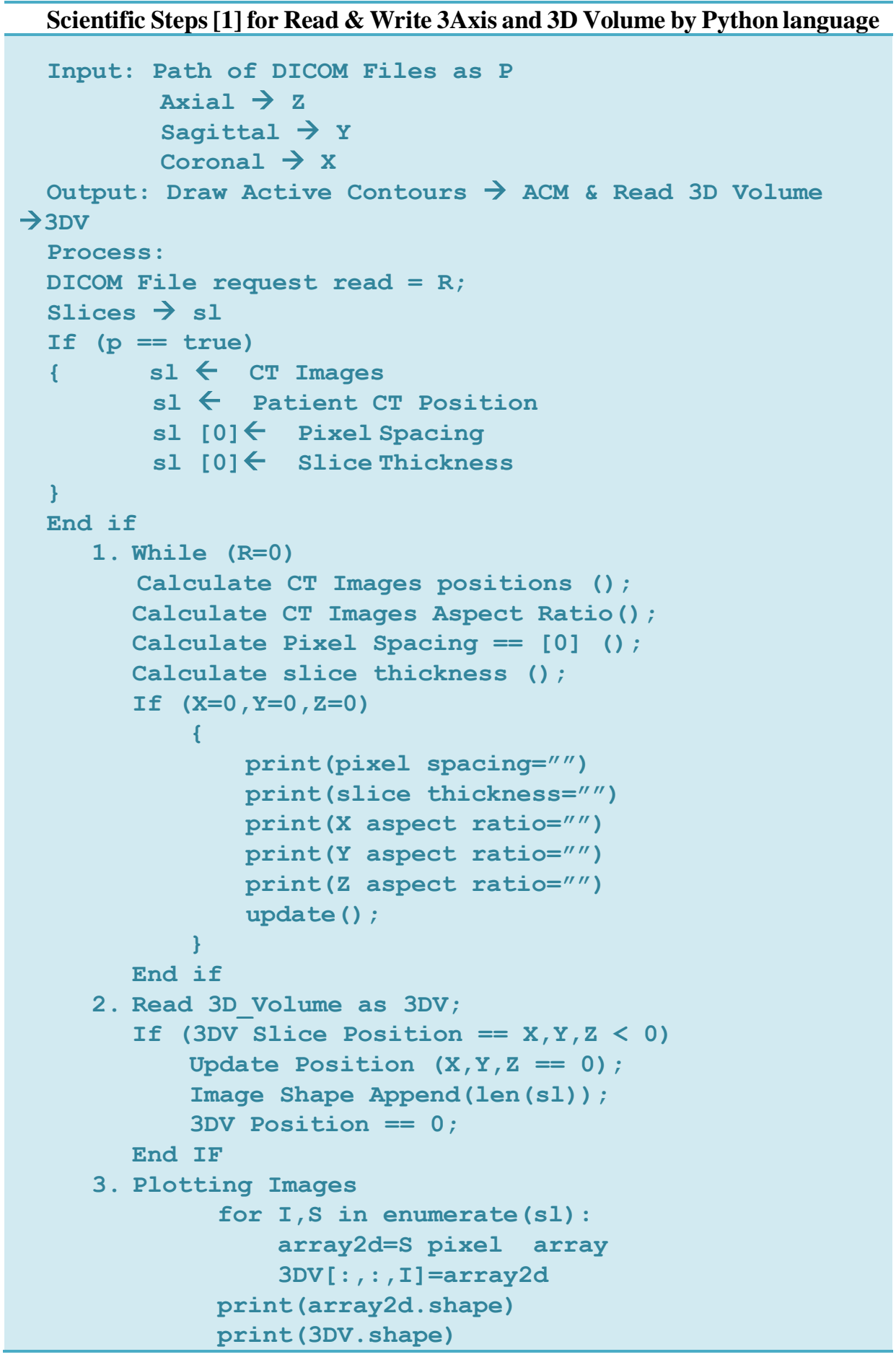



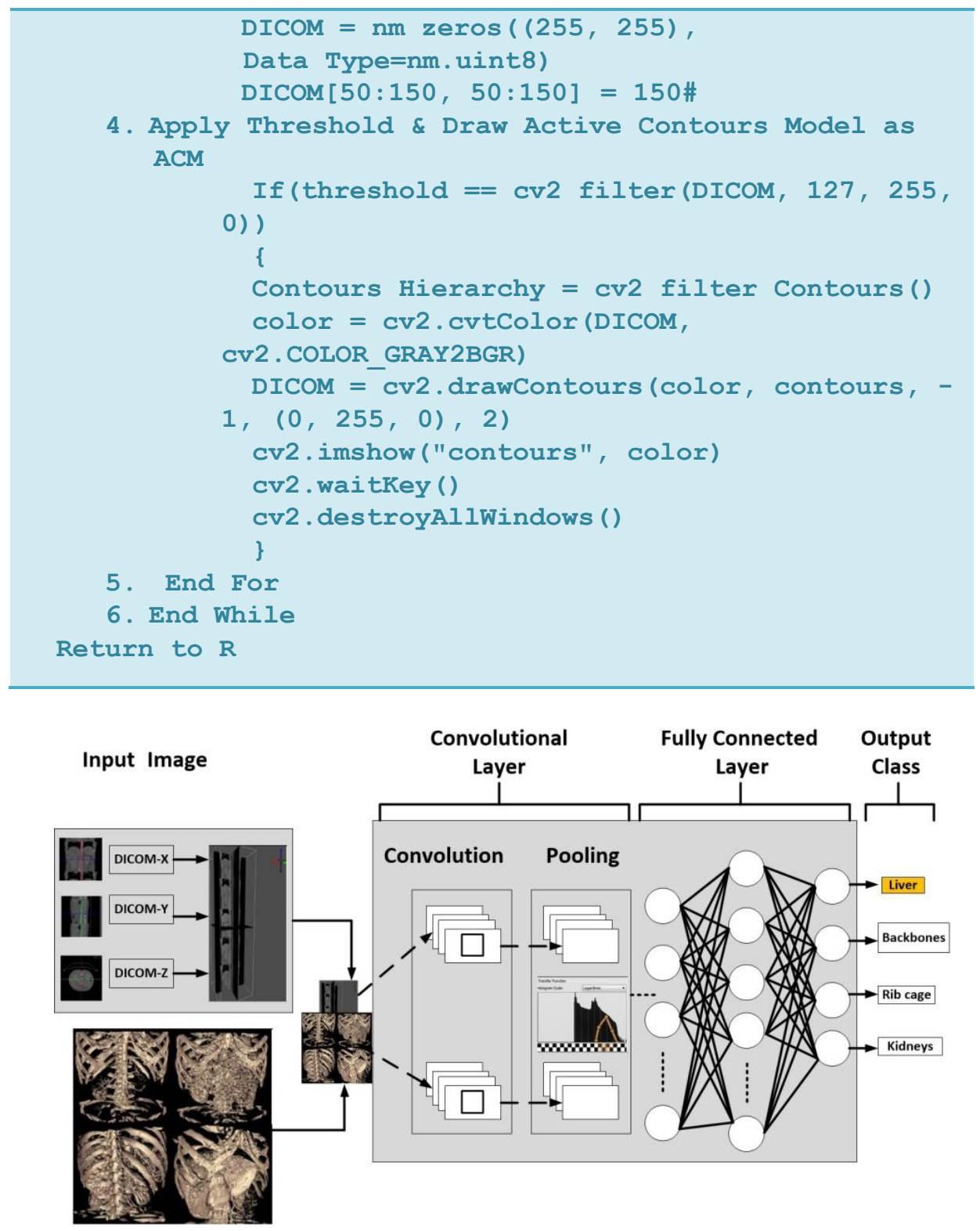

Fig. 6. $\mathrm{CNN}$ architecture

\subsection{Comparative process of medical image}

Growing the number of point light [brightness and contrast] Fig.7 (b) to improve image Accuracy \& density, as it becomes higher image quality to make it easier to convert into a 3D-model. In Scientific Steps [2] present image processing result of 
DICOM image before and after increasing point light on the image through using python and specializes in image-processing, data-visualize and data-analysis called open$\mathrm{CV}$ and NumPy library, to ensure DICOM empty from impurities and prepared to Convert.

There are no differences in the details and content of the image before and after increasing the brightness and width ratio Fig. 7, the appearance of impurities only in the borders Fig. 7(C) of the image, but it does not affect the conversion process in the three-dimensional model. The impurities are removed and the model is purified with MESHMIXER.

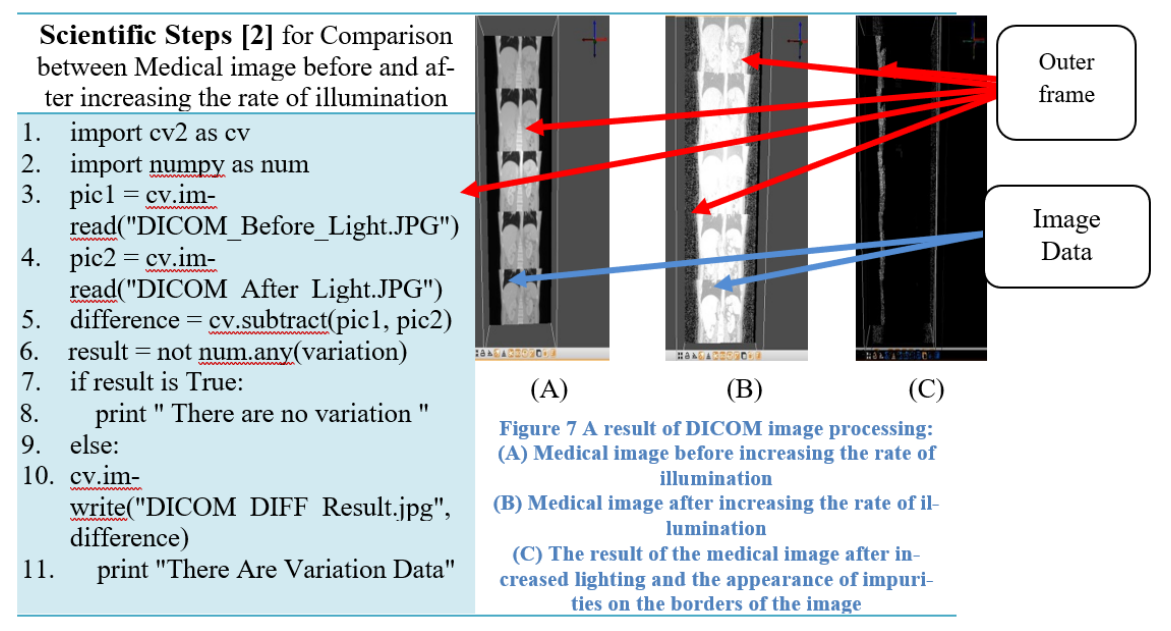

Fig. 7. A result of DICOM image processing

\subsection{Active Contour Model (ACM)}

ACM is the practical framework [28] [29] of a two-dimensional potential image diagram called the snake model and is widely used in tracking objects, recognizing shapes, image segmentation, and defining edges. A true image, knowing that snakes do not solve the problem because of the clarity of the image's features, as the best way to know in advance the shape of the contour required so that the user can interact with the user with the highest-level images, or from all other information from the surrounding image data, time and place.

To obtain a good training result, the entire liver and its surrounding pixel units must be preserved, with the missing pixel versions eliminated and the liver frame deformed so as not to cause a bad prediction if versions of many good cases are available Figure 8 .

ACM conclusion modified dependent on image and local point sentence equation terms, and expected loss that is used to train CNN to create these sanctions maps, the following Figure 8 is designed in Python to display the field of masks on the images. The proposed system of framework diagram is shown in Figure 3. 


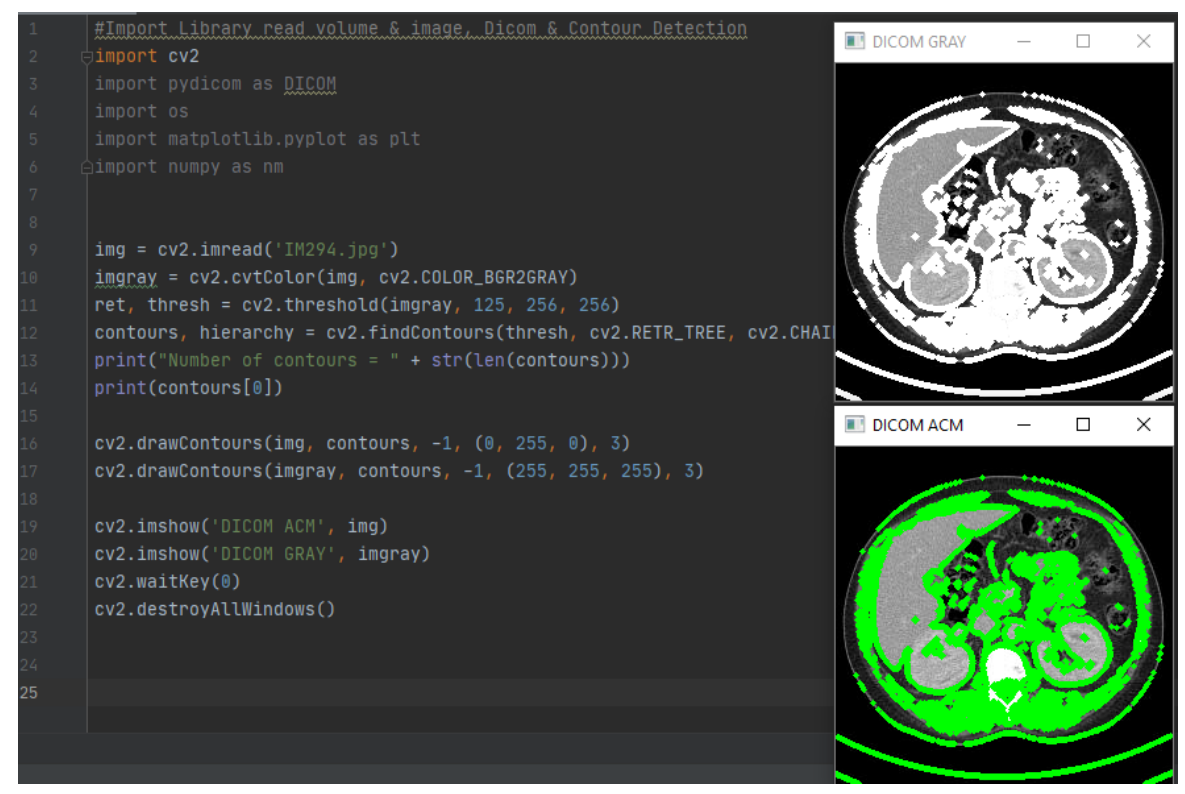

Fig. 8. Active Contour Model Mask

\subsection{Medical 2D image structure generator}

A single image is just a two-dimensional plane depicted through a projection of a three-dimensional object. Because some data is lost through the dimensions and area of the image that represent the lowest (2D) dimension from the top (3D), so no data available to originate a model-3Dimensional from 2Dimensional images Figure 9. But should generate a standard 2D CNN Structure include shape prior knowledge of the 3D object. from a single to multiple 2D-image projection mapping Figure 10, with definition a viewpoint: projection $2 \mathrm{D}$ Equal projection $3 \mathrm{D}$-axis $(\mathrm{x}, \mathrm{y}, \mathrm{z}) \&$ binary mask $(\mathrm{m})$. 


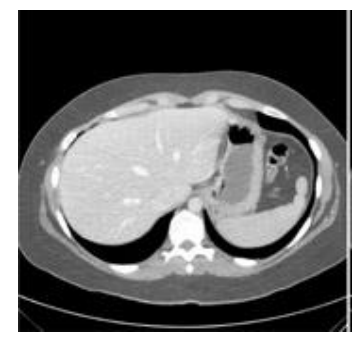

a) Full Image Without Object Detection

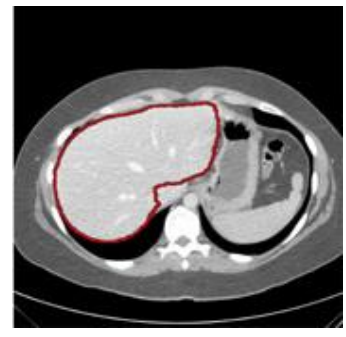

b) Liver Surrounding Pixel Units

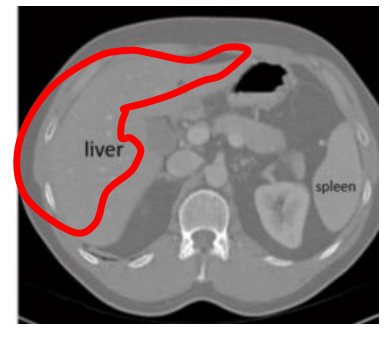

c) Single-View that's not enough data to build 3DModel

Fig. 9. Liver Active Contour

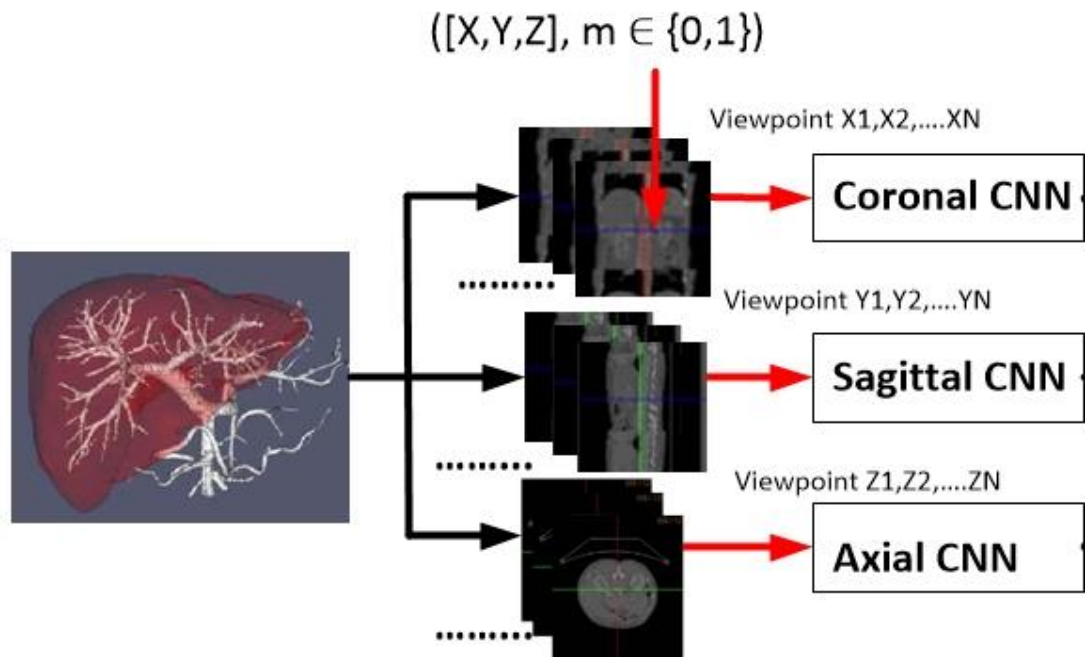

Fig. 10. 2D-Image Projection Mapping

\section{$4 \quad$ Results}

This section shows the result of active contour images from the DICOM raw image to the 3D-model. First, we show the result of the pre-processing from DICOM raw images. Each image contains edges and image size $=256$ X 256, which through adjustment and control we can create multiple results for output using Active Contour, by resizing the threshold frames and gray-scale image, shown below in table 1. 
Table 1. Results of Active Contour Model (ACM) With Threshold Mask Size

\begin{tabular}{|c|c|c|c|}
\hline$\#$ & $\begin{array}{l}\text { Threshold (ACM) } \\
\text { Color Image }\end{array}$ & $\begin{array}{c}\text { Threshold (ACM) } \\
\text { Gray Scale }\end{array}$ & Explain \\
\hline 1 & (imgray, 25, 256,20) & & $\begin{array}{l}\text { Threshold ACM }= \\
{[\mathrm{X}=25, \mathrm{Y}=256, \mathrm{X}=20]} \\
\text { Not determined of liver pixel }\end{array}$ \\
\hline 2 & (imgray, 25, 256,256) & & $\begin{array}{l}\text { Threshold ACM }= \\
{[\mathrm{X}=25, \mathrm{Y}=256, \mathrm{X}=256]} \\
\text { Determined outer body, Lost all of liver } \\
\text { pixel }\end{array}$ \\
\hline 3 & (imgray, 50, 256,256) & & $\begin{array}{l}\text { Threshold ACM = } \\
{[\mathrm{X}=50, \mathrm{Y}=256, \mathrm{X}=256]} \\
\text { Determined outer body, Lost Some of } \\
\text { liver pixel outer }\end{array}$ \\
\hline 4 & (imgray, $75,256,256)$ & & $\begin{array}{l}\text { Threshold ACM = } \\
{[\mathrm{X}=75, \mathrm{Y}=256, \mathrm{X}=256]} \\
\text { Not determined all of the liver pixel \& } \\
\text { Non-Liver }\end{array}$ \\
\hline 5 & (imgray, 100, 256,256) & & $\begin{array}{l}\text { Threshold } A C M= \\
{[X=100, Y=256, X=256]} \\
\text { determined all of liver pixel \& Non- } \\
\text { Liver }\end{array}$ \\
\hline 6 & (imgray, 125, 256,256) & & $\begin{array}{l}\text { Threshold ACM = } \\
{[X=125, Y=256, X=256]} \\
\text { determined all of the liver, Outer Non- } \\
\text { Liver \& Lost Outer Body pixel }\end{array}$ \\
\hline
\end{tabular}


Second, we can generate a lot of versions of active contour 3D-model output result, by changing the Histogram and Gaussian equation, and many of transfer function. will show all functions as shown below "table 2".

Table 2. Layers of Active Contour 3D-Model By Histogram \& Gaussian Equation

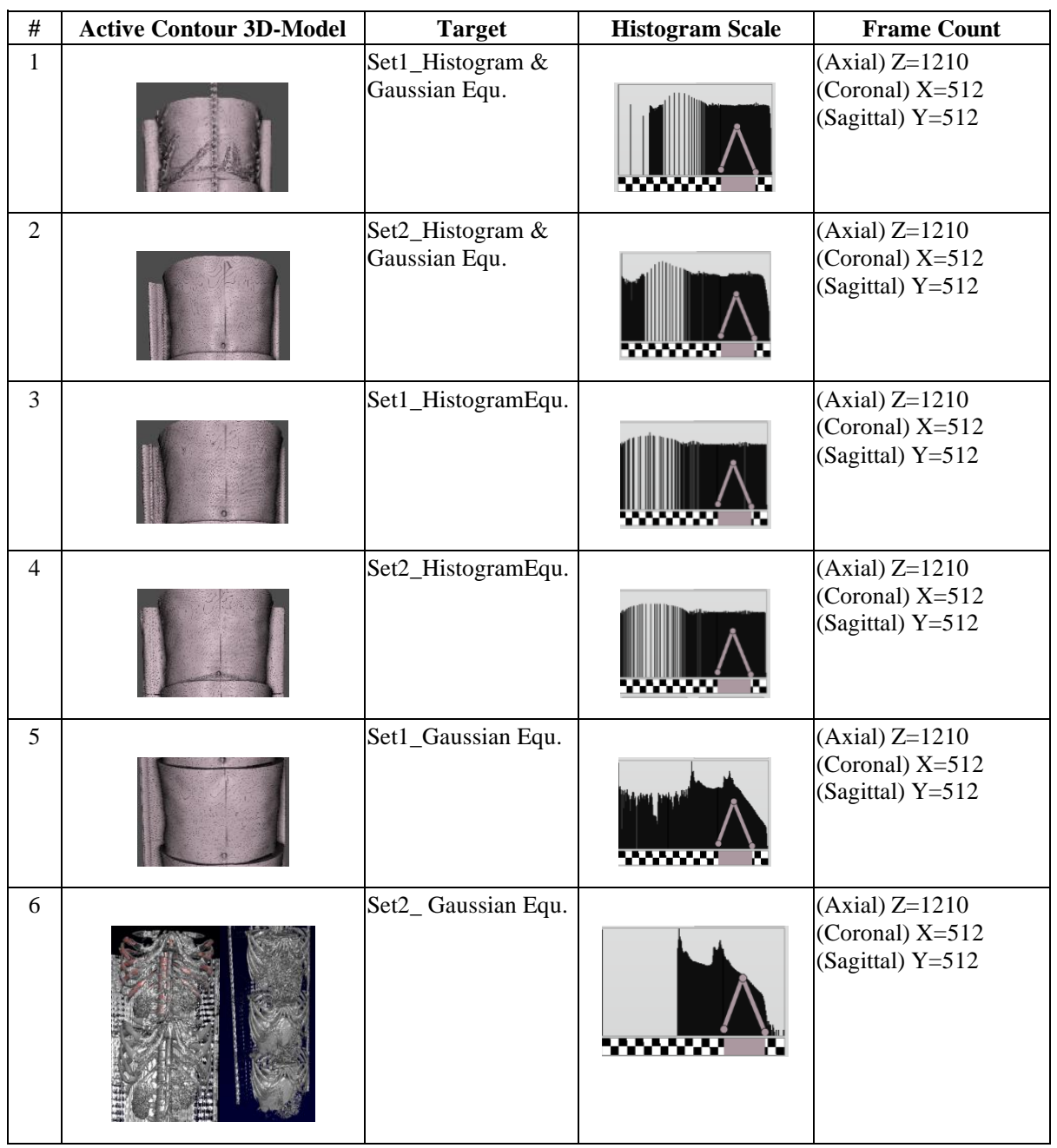

Now we can show 3D-model Deep architecture, it is through the CNN, through which the images of the three axes $\mathrm{X}, \mathrm{Y}$, and $\mathrm{Z}$ are determined and matched with a real image of the body shown above Figure 6, and the area required to be determined as shown above Figure 9 and edits the contrast using a histogram. Therefore, its prior knowledge and identification of the three dimensions of the images (three orthogonal) ( coronal $=\mathrm{X}$, sagittal $=\mathrm{Y}$, axial $=\mathrm{Z}=1$ ) to intersect them at one point and determine the liver area and convert to the 3D shape model Figure 11, Figure 12. 


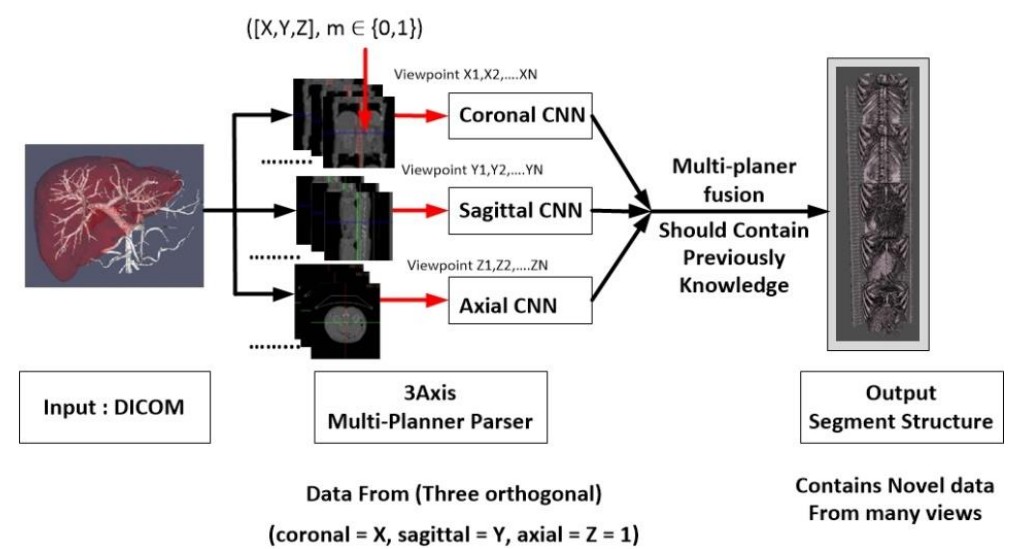

Fig. 11. Illustration of converting multi-planner to 3D-model, where the input DICOM file content 3sets of 3Axis (sagittal), corona, axial. The final 3D-model from the individual plane

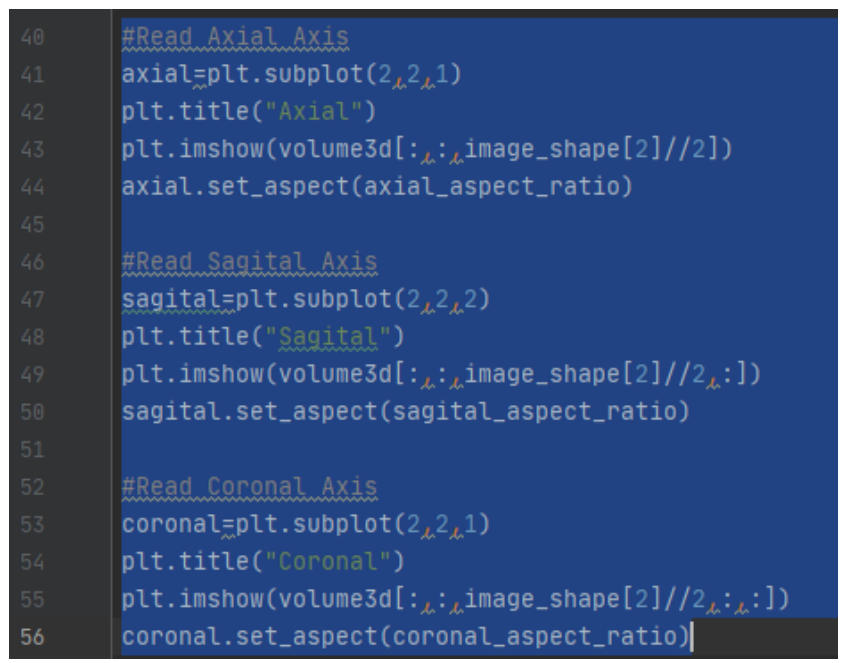

Fig. 12. Read 3-Axis [Axial, Sagital, Coronal]

To receive grate experience and training result, we should to keep versions of the active contour 3D-model which include full pixels knowledge of the liver and delete other versions which the lost pixel of a liver model, because other versions will be made more distortion when re- Sculpting \& Trimming 3D-liver model by 3d programs Figure 13. 


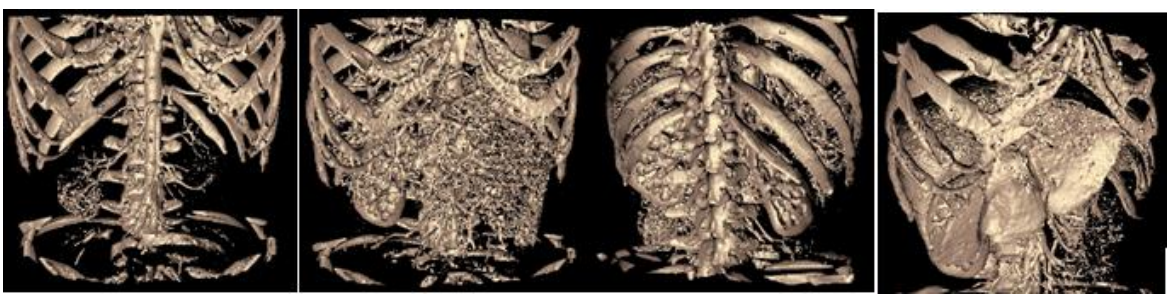

Fig. 13. 3D-liver model after re- Sculpting \& Trimming

This significant step will be to test the final simulation through a 3D-model and converted the OBJ file to a low poly object by the modeling process steps sculpt, smooth, and test the model motion in the graphics programs such as using "Adobe MESHMIXER". Therefore, in this status can be estimated outer perimeter shape stabilization and mass the liver and kidneys volume, wherever can evaluate the internal mass (Volume) by mm3 and the external perimeter (Surface Area) by mm2 Figure 14.

Now can be compared through the combination of hybrid algorithm structure (CNN, Active Contour \& Deep Multi-Planar) and seg3d2, the use of Python programming language and open-source libraries OPENCV, CV2, and NUMPY, and real results through the liver volume analysis report from MeVis Distant Services $(1731 \mathrm{ml})$, where the volume of the liver is measured in milliliters ML, and our results are MM3 $\left(1.73059 \mathrm{e}+06 \mathrm{~mm}^{3}\right)$ as shown Figure 14 , so the final results were converted into milliliters ML to ensure the correct size of the liver as shown Figure 15.

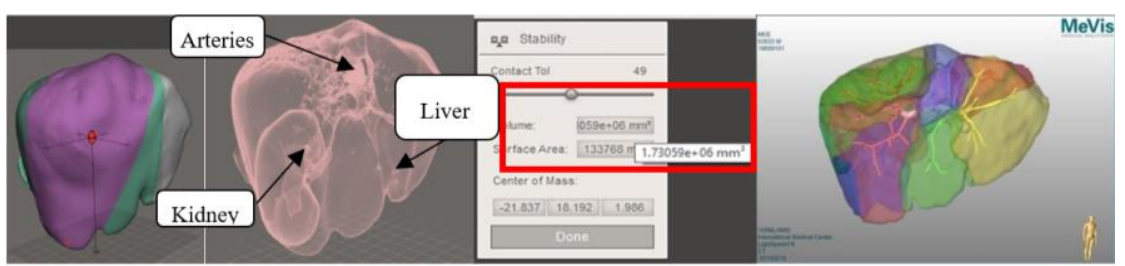

Fig. 14.OBJ file to a low poly object

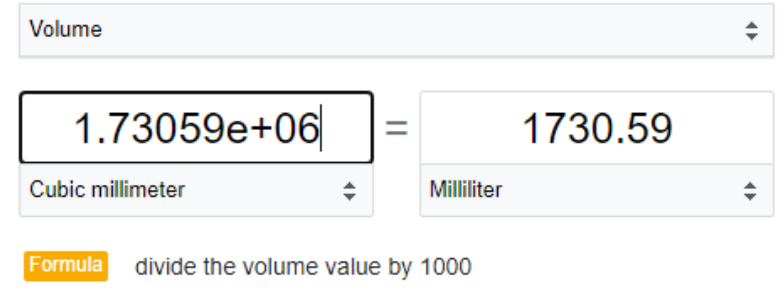

Fig. 15.Liver Volume 1731Ml (Convert from $\mathrm{mm}^{3}$ to $\mathrm{ml}$ ) 


\section{Conclusion}

We proposed in this research a simple efficient hybrid technique based on two algorithms "ACM and CNN", which has applied the power of deeply CNN to DICOM image and enhancement segmentation data files. The system achieved a high result of segmentation, each image contains edges and image size $=256 p$ X 256p, using Active Contour Model can generate multiple results for output, by resizing the threshold frames and gray-scale image and adjustment control, and generate a multi 3D model as output by changing the Histogram and Gaussian equation. In the future work, the proposed system could be utilized for detecting the Cancer and position accuracy in the Liver CT images.

\section{Abbreviations}

CNN: Convolutional Neural Networks

MR: Mixed Reality

CV: computer vision

IP: image processing

LDLT: living donor liver transplantation

DICOM: Digital Imaging and Communications in Medicine

GIS: Geographic information system

$\mathbf{M M}^{3}$ : Cubic millimeter

Ml: Milliliter

\section{$7 \quad$ References}

[1] P. Haripriya and Dr. R. Porkodi, "An Efficient Storage and Retrieval of DICOM Objects using Big Data Technologies," International Journal of Advanced Research in Computer Science, pp. 271-274, 3 March 2017.

[2] T.K.Das and P.Mohan Kumar, "Big Data Analytics: A Framework for Unstructured Data Analysis," International Journal of Engineering and Technology (IJET), 2013.

[3] Kevin Normandeau, "Beyond Volume, Variety, and Velocity is the Issue of Big Data Veracity," insidebigdata, Westborough, 2015.

[4] Ashwin Belle, Raghuram Thiagarajan, S. M. Reza Soroushmehr, Fatemeh Navidi, Daniel A. Beard and Kayvan Najarian, "BioMed Research International," Big Data Analytics in Healthcare, vol. 2015, p. 16, 16 June 2015. https://doi.org/10.1155/2015/370194

[5] "Biomed Inform Insights," Big Data Application in Biomedical Research and Health Care: A Literature Review, pp. 1-10, 82016.

[6] Mamdouh, Rafeek \& El-Bakry, Hazem \& Riad, Alaa el-din \& El-Khamisy, Nashaat. (2020). Converting 2D-Medical Image Files "DICOM" into 3D-Models, based on Image Processing, and Analysing Their Results with Python Programming. WSEAS Transactions on Computers. 19. 10-20. https://doi.org/10.37394/23205.2020.19.2

[7] Cheng, H. D., Jiang, X. H., Sun, Y., \& Wang, J. (2001). Color image segmentation: advances and prospects. Pattern recognition, 34(12), 2259-2281. https://doi.org/10.1016/s0031$\underline{\text { 3203(00)00149-7 }}$ 
[8] S. K. Pal et al., \A Review on Image Segmentation Techniques," Pattern Recognition, 29, 1277-1294, 1993.

[9] K. S. Fu and J. K. Mui, \A Survey on Image Segmentation," Pattern Recognition, 13, 3-16, 1981.

[10] E. M. Riseman and M. A. Arbib, IComputational Techniques in the Visual Segmentation of Static Scenes", Computer Vision, Graphics and Image Processing, 6, 221-276, 1977. https://doi.org/10.1016/s0146-664x(77)80028-2

[11] S, Prasantha. (2020). Image Segmentation Algorithms on Digital Signal Processor. 8. 34223427.

[12] Das, Shubhajoy. (2020). Medical Image Segmentation. Journal of Mechanics of Continua and Mathematical Sciences. 15. 10.26782/jmcms.2020.07.00009.

[13] Wu, Jianxin. And LAMDA Group (2020). The Normal Distribution. National Key Lab for Novel Software Technology. https://cs.nju.edu.cn/wujx/teaching/13_Gaussian.pdf

[14] Bücking, T. M., Hill, E. R., Robertson, J. L., Maneas, E., Plumb, A. A., \& Nikitichev, D. I. (2017). From medical imaging data to 3D printed anatomical models. PloS one, 12(5), e0178540. https://doi.org/10.1371/journal.pone.0178540

[15] Amer, Khaled \& El-Khamisy, Nashaat \& Mamdouh, Rafeek \& Riad, Alaa el-din. (2018). Survey on Virtual Reality, Augmented Reality, and Mixed Reality Techniques for Liver Surgical Operations and Training.

[16] Mamdouh, Rafeek. (2020). Using Azure to construct recent architecture for visual training in real-time. Indian Journal of Science and Technology. 13. 2355-2370. https://doi. org/10.17485/ijst/v13i19.649

[17] Rafeek Mamdouh, Hazem M. El-Bakry, Alaa Riad, and Nashaat El-Khamisy, "Improve Speed Real-Time Rendering in Mixed Reality HOLOLENS during Training" International Journal of Advanced Computer Science and Applications (IJACSA), 11(3), 2020. https://doi.org/10.14569/ijacsa.2020.0110339

[18] Yamashita, R., Nishio, M., Do, R. K. G., \& Togashi, K. (2018). Convolutional neural networks: an overview and application in radiology. Insights into Imaging, 9(4), 611-629. https://doi.org/10.1007/s13244-018-0639-9

[19] Phung, \& Rhee, (2019). A High-Accuracy Model Average Ensemble of Convolutional Neural Networks for Classification of Cloud Image Patches on Small Datasets. Applied Sciences. 9. 4500. 10.3390/app9214500. https://doi.org/10.3390/app9214500

[20] Minaee, Shervin \& Boykov, Yuri \& Porikli, Fatih \& Plaza, Antonio \& Kehtarnavaz, Nasser \& Terzopoulos, Demetri. (2020). Image Segmentation Using Deep Learning: A Survey. https://doi.org/10.1109/tpami.2021.3059968

[21] Moeskops, P., Wolterink, J. M., van der Velden, B. H., Gilhuijs, K. G., Leiner, T., Viergever, M. A., \& Išgum, I. (2016, October). Deep learning for multi-task medical image segmentation in multiple modalities. International Conference on Medical Image Computing and Computer-Assisted Intervention (pp. 478-486). Springer, Cham. https://doi.org/10.1007/ 978-3-319-46723-8_55

[22] Bao, S., \& Chung, A. C. (2018). Multi-scale structured CNN with label consistency for brain MR image segmentation. Computer Methods in Biomechanics and Biomedical Engineering: Imaging \& Visualization, 6(1), 113-117. https://doi.org/10.1080/21681163.2016.1182072

[23] Yamashita, R., Nishio, M., Do, R. K. G., \& Togashi, K. (2018). Convolutional neural networks: an overview and application in radiology. Insights into Imaging, 9(4), 611-629. https://doi.org/10.1007/s13244-018-0639-9

[24] Nguyen-Thanh-Minh, N., \& Tran-Van-Hoang, T. (2020). Liver CT Image Processing and Diagnosing Using Artificial Neural Networks And MATLAB. In Proceedings of International Sym (Vol. 3, pp. 79-88). https://doi.org/10.29007/3dj7 
[25] Mortazi, A., Karim, R., Rhode, K., Burt, J., \& Bagci, U. (2017, September). CardiacNET: Segmentation of left atrium and proximal pulmonary veins from MRI using multi-view CNN. International Conference on Medical Image Computing and Computer-Assisted Intervention (pp. 377-385). Springer, Cham. https://doi.org/10.1007/978-3-319-66185-8_43

[26] Mortazi, A., Burt, J., \& Bagci, U. (2017, September). Multi-planar deep segmentation networks for cardiac substructures from MRI and CT. In International Workshop on Statistical Atlases and Computational Models of the Heart (pp. 199-206). Springer, Cham https://doi.org/10.1007/978-3-319-75541-0_21

[27] Zhou, Y., Wang, Y., Tang, P., Bai, S., Shen, W., Fishman, E., \& Yuille, A. (2019, January). Semi-supervised 3D abdominal multi-organ segmentation via deep multi-planar co-training. In 2019 IEEE Winter Conference on Applications of Computer Vision (WACV) (pp. 121140). IEEE. https://doi.org/10.1109/wacv.2019.00020

[28] Kalyani, C., Ramudu, K., \& Reddy, G. R. (2020). Enhancement and Segmentation of Medical Images Using AGCWD and ORACM. International Journal of Online \& Biomedical Engineering, 16(13).

[29] BR, N., HS, C., \& MS, D. (2020). Oral Malignancy Detection Using Color Features from Digital True Color Images. International Journal of Online \& Biomedical Engineering, 16(14).

\section{Authors}

Rafeek Mamdouh Tawfiq, corresponding author, Ph.D. candidate, Faculty of Computer and Information System, Mansoura University. Egypt. Received BSC of (MIS) Management Information System in 2006 from Shorouk academy, Cairo, Egypt, and MSc in computer graphics and information system, specializing Visualization \& 3DMotion Capture, Sadat Academy 2013, Cairo, Egypt. He was worked at Brainwaves SME system analysis and design of simulation and visualization department and $3 \mathrm{~d}$ graphic designer (2007-2011), his worked supervisor of motion capture department at Arab Gulf Production and NEWTECH media production in $(2014,2017)$. He is mainly interested in machine learning applications, IoT, Algorithms, 3D Visualizations and Knowledge discovery in Azure. Rafeek works as an assistant lecturer at the British University and Shorouk Academy and other institutes from 2012 to 2017. Rafeek is an Assistant Lecturer of Multimedia and Graphics Dept., at faculty of filmmaking and performing arts, BADR University, Cairo, Egypt to present. Rafeek has published papers in international journal indexed ISI, Scopus and web of Sciences. (Email:rafeekmty@ hotmail.com).

Nashaat El-Khameesy El-Ghitany, is Prof. of Computers \& Information System, his worked the Dean Sadat Academy for Management Sciences (SAMs), Cairo, Egypt. Dr. Nashaat graduated BSc in Elec. Eng the Military Technical College, (MTC) Cairo, Egypt. MSc. in computers, Faculty of Eng. Cairo-University 1980. He earned his PhD in Electrical Eng, Ohio State Univ., USA, 1988. He started his academic career in MTC, his worked University Council member, Director of R\&D, Dean of college of Port Said, Tanta, and Mansoura branches, SAMs 2000-2007. Dr Nashaat was awarded the Spanish Royal Academy Award 2011, Best of AMSE Award (Association for the Advancements in Modeling and Simulation for Enterprises), DR Nashaat has over 120 publications in international journals and conferences while also supervised over $100 \mathrm{MSc}$ and 
$\mathrm{PhD}$ dissertation, and he's the author of many text books in areas of informatics, e business and health informatics. His current research interest includes business intelligence, e-business \& e-gov., performance enhancement, and cyber security. he's the Vice-president of the Int. Association for Advanced Modeling and Simulation for Enterprises (AMSE) (www.amse-modeling.org), Barcelona-Paris. He's also the co-founder of the Egyptian Association for Computers and Information Technology (ESIS-ACT) and the ICI committee (Texas A\&M-USA, Esterhazy Academy-Hungary, Open Univ.-Malaysia, Agri-Media). He also addressed many keynotes in many events locally and internationally. (Email: wessasalsol@gmail.com).

Dr. Khaled Amer is best known for his exceptional work in the Military Medical Services, as the "Chief Consultant for Liver Transplantation and Liver Research". In addition, he is also currently holding other significant positions in other institutes, in Cairo, Egypt, such as, being the "Professor of General Surgery" \& the "General Surgery Board Member" in the Military Medical Academy, "Consultant of Hepatobiliary and Live Transplant Surgeon in the Gastrointestinal Surgery Department" at the International Medical Center, and above all the CEO of the Egyptian Center for Research and Regenerative Medicine. Dr. Amer previously held other important positions, some of which included, the "Dean" at Armed Forces College of Medicine. Dr. Amer was graduated from the Faculty of Medicine at Ain Shams University, Cairo, Egypt in 1983 and received his Medical Doctorate of Surgery, on November 2000, from the Faculty of Medicine at Ain Shams University, and his Fellowship of the Royal College of Surgeons England, on October 2014. During his work in "Living Donor Liver Transplantation" (LDLT), Dr. Amer had the privilege to collaborate with multiple foreign experts/institutes in his field of work. He worked on the graft selection in LDLT at Kings College, UK. In Kyoto University, Japan, and Kobe Institute for Research \& Innovations, Japan. Aside from his impressive work and educational background, Dr. Amer is also an active member in some of the major professional societies, which include, International Society of Liver Transplantation (ISLT), International College of Surgeon (ICS), European Association of Endoscopic Surgery (EAES), Society of American Gastrointestinal \& Endoscopic Surgeons (SAGES), Egyptian Society of Surgeons (ESS) and last but not least, a member of the founder board of the Egyptian Society of Surgeons Group of Hepatobilary and Pancreatic Surgeons (EGHBPS). dramertx@gmail.com

A.M. Riad is a professor at Faculty of Computers and Information, Mansoura University. He has received BS, MS, and PhD. in Electrical Engineering in 1982, 1988, and 1992 respectively, from Mansoura University, Faculty of Engineering, Egypt. Dr. Riad worked as the dean of the faculty of Computers and Information, Mansoura University. In addition, he worked as the chairman of information systems department, Faculty of Computers and Information. His research interests include expert systems, cloud computing smart cities and big data analysis. Dr. Riad has authored and coauthored many research papers in published journals. He also has supervised many Master and Doctorate studies. Reviewer in several national and international scientific journals. Information about publications, activities and personal blog are available at the Web site: http://www.amriad.com or http://www.amriad.name.eg. (Email: $\underline{\operatorname{amriad} 2014}$ @ gmail.com). 
Hazem El-Bakry (Mansoura, EGYPT 20-9-1970) received B.Sc. degree in Electronics Engineering, and M.Sc. in Electrical Communication Engineering from the Faculty of Engineering, Mansoura University - Egypt, in 1992 and 1995 respectively. Dr. El-Bakry received Ph. D degree from University of Aizu-Japan in 2007. Currently, he is full professor at the Faculty of Computer Science and Information Systems Mansoura University-Egypt. He is the head of Information Systems Dept. His research interests include neural networks, pattern recognition, image processing, biometrics, cooperative intelligent systems and electronic circuits. In these areas, he has published many papers in major international journals and refereed international conferences. According to academic measurements, now the total number of citations for his publications is 3698 and the H-index of his publications is 30. Dr. El-Bakry has the United States Patent No. 20060098887, 2006. Furthermore, he is associate editor for journal of computer science and network security (IJCSNS) and journal of convergence in information technology (JCIT). In addition, he is a referee for IEEE Transactions on Signal Processing, Journal of Applied Soft Computing, the International Journal of Machine Graphics and Vision, the International Journal of Computer Science and Network Security, Enformatika Journals, WSEAS Journals and many different international conferences organized by IEEE. Moreover, he has been awarded the Japanese Computer and Communication prize in April 2006 and the best paper prize in two conferences cited by ACM. He has also been awarded Mansoura university prize for scientific publication in 2010 and 2011. Dr. El-Bakry has been selected in who Asia 2006 and BIC 100 educators in Africa 2008. (Email: elbakry@ mans.edu.eg).

Article submitted 2021-01-15. Resubmitted 2021-03-11. Final acceptance 2021-03-20. Final version published as submitted by the authors. 